\title{
Lab Classes in Chemistry Learning an Artificial Intelligence View
}

Margarida Figueiredo ${ }^{1}$, M. Lurdes Esteves ${ }^{1}$, José Neves ${ }^{2}$, and Henrique Vicente ${ }^{1}$

${ }^{1}$ Departamento de Química e Centro de Química de Évora,

Universidade de Évora, Évora, Portugal

\{mtf, m4233, hvicente\}@uevora.pt

${ }^{2}$ Departamento de Informática, Universidade do Minho, Braga, Portugal jneves@di.uminho.pt

Abstract. The teaching methodology used in lab classes in Chemistry Learning was studied for a cohort of 702 students in the $10^{\text {th }}$ grade of Portuguese Secondary Schools. The k-Means Clustering Method, with k values ranging between 2 (two) and 4 (four), was used in order to segment the data. Decision Trees were used for the development of explanatory models of the segmentation. The results obtained showed that the majority of the answerers considered that experimentation is central on Chemistry learning. The results also showed that the significance of research in Chemistry learning is strongly dependent on the students' involvement in lab work.

Keywords: Artificial Intelligence, Chemistry Learning, Decision Trees, k-Means, Lab Classes, Lab Work.

\section{Introduction}

In the Society of the XXI century, the scientific and technological knowledge stand, unquestionably, to its development. In order to surpass this challenge, the teaching of Sciences, in general, and of Chemistry, in particular, points to general objectives, independently of the level of education that must be attained, i.e., in the one hand the acquisition of a scientific attitude, the development of reasoning skills, analytical capabilities, critical and inventive thinking should be highlighted. On the other hand, the importance and the implication of Science in Society should also be valorised. The achievement of these goals depends more on a solid scientific background than on a very specialized teaching or on the acquisition of technical knowledge, swiftly out-dated. Thus, in the future, the emphasis in teaching must be placed on developing skills and not on the transmission of knowledge. The student cannot be a passive element in the teaching-learning process. Instead, the student must be an active and proactive element in his/her own education.

Undeniably, nowadays, there is a lack of interest among students in learning Chemistry [1]. This fact may be related with the kind of education provided. Indeed, there is a tendency to reduce the teaching of Chemistry to its formal aspects, i.e., to a 
mathematical overview of the theme. This trend may be blameable for Chemistry being regarded as a hard subject to tackle and, therefore, answerable for the decrease of the number of students attending Chemistry courses. However, Chemistry, like other Sciences, has a powerful instrument to promote meaningful learning - The Lab Work.

In the traditional instruction of the sciences predominate the lectures with the aim to "deliver" ideas or information from the teacher to the students [2]. However, for the study of most topics in science this method is inappropriate, and the theoretical lectures are not enough. Indeed, lab work has a long-established and fundamental role in Chemistry teaching, something that has been acknowledgeable for the last three centuries $[3,4,5,6,7,8,9,10]$. Nevertheless, only since the nineteenth century, lab work has been officially included in the curricula of Sciences, regardless of the denomination adopted. However, doubts were sometimes raised about its importance as a means for promoting significant learning of Chemistry [11,12]. What function should lab work play in such a teaching process? Should lab work continue to be carried out in a traditional manner or should it assume other characteristics according to other roles?

Although it is not a consensual opinion, many authors consider that lab work represents a fundamental resource in the teaching of Science [13], and different types of lab work with different objectives, leading to dissimilar learning outcomes, should be carried out [4] [14]. The methodology that most limits the role of the student is to carry out demonstrations done by the teacher, followed by an explanation of the practises, in which the student merely observes. Although this methodology is very limited in terms of learning, it is still used in some schools, due to the shortage of material resources. In these cases the students have no opportunity to develop any of the skills usually presented as advantages to adopt the realization of lab work in schools. A second type of practice consists in carrying out the lab work by students according to recipes executed in succession. Students focus their thoughts on finishing one step after another and many times they do not develop a deeper understanding of the experiments. For many students lab work means just working, handling laboratory equipment, not including, generally, the development and the understanding of scientific thinking [4]. In a third type of methodology the students perform lab work autonomously by planning and executing the work. According to Killerman [15] the students that conducted experiments by themselves were very enthusiastic about work and more motivated for the subject.

Some studies confirm that laboratory based learning quality has increased as students have an active role in the process of gaining knowledge [4] $[16,17]$. There are several methods that allow one to explore this type of learning, like class research seminars, problem based learning, case studies, project-based learning, role playing, cooperative and cooperation learning, group debate, development of mind maps, experience based learning, just to name a few.

In this study we intend to find a response to the following question: Does the achievement of lab work by students in the classes of chemistry increase their learning?

In the present study, Knowledge Discovery from Databases (KDD) was the strategy followed to treat results. The designation KDD refers to a process that involves the identification and recognition of patterns in a database, in an automatic way, i.e. obtaining relevant, unknown information, that may be useful in a decision making process, without a previous formulation of hypothesis $[18,19]$.

In terms of analysis of data, another question may be formulated: Will the Date Mining approach allow a deeper analysis of the results when compared with a conventional approach like the analysis of frequencies of responses?

\section{Methods}

2.1 Sample

A total of 702 students from the $10^{\text {th }}$ grade were enrolled in this study. They come from secondary schools located in the north of Portugal (district of Bragança), centre (district of Castelo Branco), and south (districts of Beja, Évora and Faro). The districts of Beja, Bragança, Castelo Branco and Évora are situated in the interior region of the country, while the remaining one is located in the coastal line. Table 1 shows the characterization of the sample in terms of age, gender, grade and district. A perusal of Table 1 reveals that $45.0 \%$ of students are male and $55.0 \%$ are female. Concerning the students' age, $83.3 \%$ of them did not exceed 16 (sixteen) years old, which suggests that the grade repetition levels are low. The geographical location seems not to influence the results, since the percentage of this class of students varies between $79.9 \%$ (district of Bragança) and $88.5 \%$ (district of Castelo Branco).

\subsection{Procedure}

In order to fulfill the goals defined so far, an adaptable tool to data collection was essential, with the potential to be used in a wide geographical area and on time $[20,21]$. After considering and analyzing the advantages and limitations intrinsic to the various techniques available, a practice based on the inquiry by questionnaire was chosen [22]. This instrument has a well-defined structure and allows for the information conveyed in a conversion reported by the answerers, to be considered in a quantitative fashion. The questions included in the questionnaire were planned, on the one hand, to allow for the gathering of information on the learning methodologies followed in the lab classes and, on the other hand, to scrutinize the influence of such methodologies in Chemistry learning. The questions were organized into 3 (three) main categories, namely the characterization of the lab classes of Chemistry, the characterization of post-lab work, and the importance of experimentation in Chemistry learning. The former group include questions such as Who does the lab work?; How are the students organized in the lab classes?; and Which is the basis of the lab work?. The second one comprises the question What type of post-lab work is done?; and the latest includes the question What is the importance of experimentation on the learning of Chemistry?. In each question students may choose one of the proposed answers. Taken as example the last question, the options are Very high, High, Moderate, Low and Very low. For the remaining questions the answers options may be found in Figure 1. 
Table 1. Assessment sample in terms of age, gender, grade and district

\begin{tabular}{|c|c|c|c|c|c|c|c|}
\hline \multirow{2}{*}{ District } & \multicolumn{5}{|c|}{ Age } & \multicolumn{2}{|c|}{ Gender } \\
\hline & $<15$ & 15 & 16 & 17 & $>17$ & F & M \\
\hline $\mathrm{Beja}^{\mathrm{c},{ }^{*}}$ & 0 & 71 & 52 & 17 & 10 & 89 & 61 \\
\hline Bragança, ${ }^{a, "}$ & 0 & 43 & 68 & 21 & 7 & 75 & 64 \\
\hline Castelo Branco ${ }^{\mathrm{b}, *}$ & 0 & 62 & 46 & 11 & 3 & 64 & 58 \\
\hline Évora ${ }^{c, *}$ & 1 & 52 & 43 & 15 & 9 & 66 & 54 \\
\hline Faro ${ }^{c,+}$ & 0 & 89 & 58 & 8 & 16 & 92 & 79 \\
\hline Numeric & 1 & 317 & 267 & 72 & 45 & 386 & 316 \\
\hline Percentage total & $\approx 0$ & 45.3 & 38.0 & 10.3 & 6.4 & 55.0 & 45.0 \\
\hline
\end{tabular}

The validation of the questionnaire respects the practices recommended by Bell [23]. Subsequently, the questionnaire was evaluated by a group of experts that suggested some amendments. As soon as these revisions where done, the questionnaire was applied to a small group of students not included in the sample, to check interna consistency of the questionnaire and to identify possible difficulties in the interpretation of the questions.

\subsection{Data Analysis}

Before attempting the Data Mining modelling, the data was pre-processed. The incomplete questionnaires (missing an answer to at least one question) or those that exhibited invalid answers (two or more options marked for the same question) were discarded.

Clustering Analysis. Clustering is one of the most appropriate assignments on the data-mining phase for uncovering groups and identifying interesting distributions and patterns in data. The $\mathrm{k}$-means clustering method $[18,19]$ is one of the most efficient and popular partitioning clustering algorithms. Clustering models focus on identifying groups of similar objects, and label the objects according to the group to which they belong. This is done without the use of prior knowledge about the groups and their characteristics. These models are often referred to as unsupervised learning models, since there is no external standard by which to judge the models' performance. Their value is determined by their ability to capture thought-provoking grouping in the data and provide useful descriptions of those assemblages.

The basic idea in the k-means clustering method is to try to discover $\mathrm{k}$ clusters, according to the requirements:

- each cluster must contain at least one object; and

- each object must belong to exactly one cluster.

The k-means algorithm input parameters stand for the number of clusters, $\mathrm{k}$, and a data set, $\mathrm{D}$, with $\mathrm{n}$ objects. As soon as the algorithm is enforced, it selects, randomly, $k$ points that denote the initial centers of the clusters, being the objects assigned to the cluster to which they are akin, which is done according to the Euclidean distance be-

the number of clusters, $k$, that here ranged from 2 (two) to 4 (four). $\mathrm{k}$ values greater than 4 (four) were also tested. In these cases some clusters comprise a reduced number of objects and, therefore, these models are not presented in this work.

Decision Trees and Extraction Rules. In order to generate an descriptive model for the clusters so far shaped, Decision Trees (DTs) were used. DTs have many attractive features, such as allowing human interpretation, and hence making it possible for a decision maker to gain insights into what factors are critical for a particular classification process. DTs adopt a branching structure of nodes and leaves, where the knowledge is hierarchically organized. Each node tests the value of a feature, while each leaf is assigned to a class label. The basic strategy employed to generate DTs is the so-called recursive partitioning or the divide-and-conquer approach to problem solving. It works by partitioning the examples by choosing a set of conditions on an independent variable, such that an error on the dependent variable is minimized within each group. The process continues recursively inside each subgroup until certain conditions are met, like the ones where the error cannot be further reduced (e.g. where al instances in a group belong to the same class) [19]. Sometimes, it is useful to build a rule-based classifier by extracting IF-THEN rules from the DTs. The rule is created at each path, from the root to a leaf node. Each splitting criterion along a given path is logically ANDed to form the rule antecedent (the IF part). The leaf node holds the class prediction, forming the rule consequent (the THEN part).

The DT algorithm used in this study was the J48 as implemented in WEKA [25] This J48 implements the $8^{\text {th }}$ revision of the commonly known C4.5 algorithm. A description of the J48 algorithm can be found in Witten \& Frank [18].

\section{Results}

\subsection{Database}

Building upon the questionnaires answers, a database was built with a total of 5 (five) fields, with 702 (seven hundred and two) valid records. The frequencies of answers to each inquiry are presented in Fig. 1, and expose that only a few answerers indicated that is the teacher that does the lab work. A significant majority of the answerers also claim that the lab work is developed from experimental guidelines, and that the postlab work consists on the elaboration of written reports. The analysis of Fig. 1 also denotes that the overwhelming majority of the answerers acknowledged that experimentation is essential to learn Chemistry. 

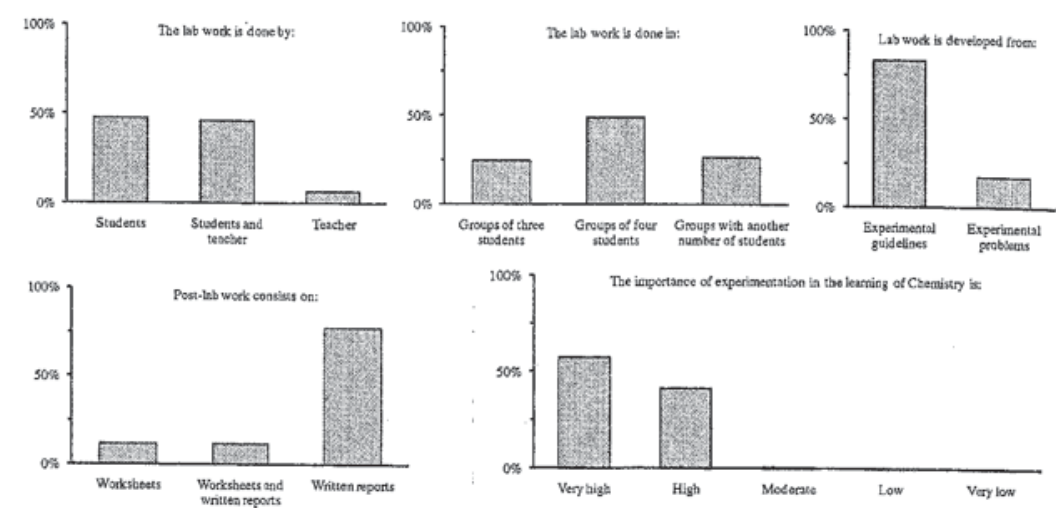

Fig. 1. Frequencies of the answers given to each question by the answerers

In order to examine if the answers to the questionnaires are influenced by the geographical location of the schools, an attempt was made to relate the student's replies to the questions presented above, within the various districts. The results show that the answers are not influenced by the geographical location of the schools and, therefore, may be analyzed together.

\subsection{Segmentation Models - Assessment and Interpretation}

Regarding the responses obtained in the questionnaire, the analysis of Table 2 shows that the $k=3$ and the $k=4$ clustering models are quite similar. The main difference is the division of cluster 2 (two) of the $k=3$ clustering model into cluster 2 (two) (with 140 objects) and cluster 4 (four) (with 172 objects), into the $k=4$ clustering model. Table 2 further reveals that cluster 1 (one) includes only students who claim that the lab classes are developed from tentative situations. Regarding cluster 3 (three), this is made upon students that assert that exclusively themselves do the lab classes. The splitting of cluster 2 (two) (model of three clusters) into two clusters (model of four clusters), enable to group a part of the students that reported that the lab classes are done sometimes by themselves and occasionally by the teacher, into cluster 4 (four). With respect to the model of two clusters, a glance of Table 2 shows that cluster 1 (one) was formed by the students that claim that the classes lab are done always by themselves, while cluster 2 (two) comprises the students that reported the opposite.

\subsection{Explanatory Models of Segmentation}

In order to generate explanatory models of segmentation (i.e. seeking to establish rules for assigning a case to a cluster), Decision Trees (DTs) were used. To ensure statistical significance of the attained results, 20 (twenty) runs were applied in all tests, being the accuracy estimated using the holdout method. Thus, in each simulation, the available data is randomly divided into 2 (two) mutually exclusive partitions, i.e., the training set, with two-thirds of the available data and used to construct the models, and the test set, with the remaining of the examples being used after training, in order to compute the accuracy values [18]. The DTs obtained are shown in Fig. 2. The rule to assign a case to cluster 1 (one) is "The basis of lab work is Experimental Problems". To allocate a case to cluster 2 (two) there are twofold rules. The former sets that The basis of lab work is Experimental Guidelines and the lab work is done by Teacher, while the latest sets that The basis of lab work is Experimental Guidelines and the lab work is done sometimes by the Students and sometimes by the Teacher. Finally, the rule to assign a case to the cluster 3 (three) stands for The basis of lab work is Experimental Guidelines and the lab work is done by the Students.

A common tool for classification analysis is the coincidence matrix (also known as confusion matrix) $[18,19]$, a matrix of size $\mathrm{L} \times \mathrm{L}$, where $\mathrm{L}$ denotes the number of possible classes. The matrix is built by matching the values predicted by the model (rows) with the actual values (columns). The coincidence matrixes, presented in Table 3 , reveal that the accuracy of the DT displayed in Fig. 2 is $100 \%$ for both training and test sets.

Table 2. Answers obtained on the questionnaire split by issues and by clustering models

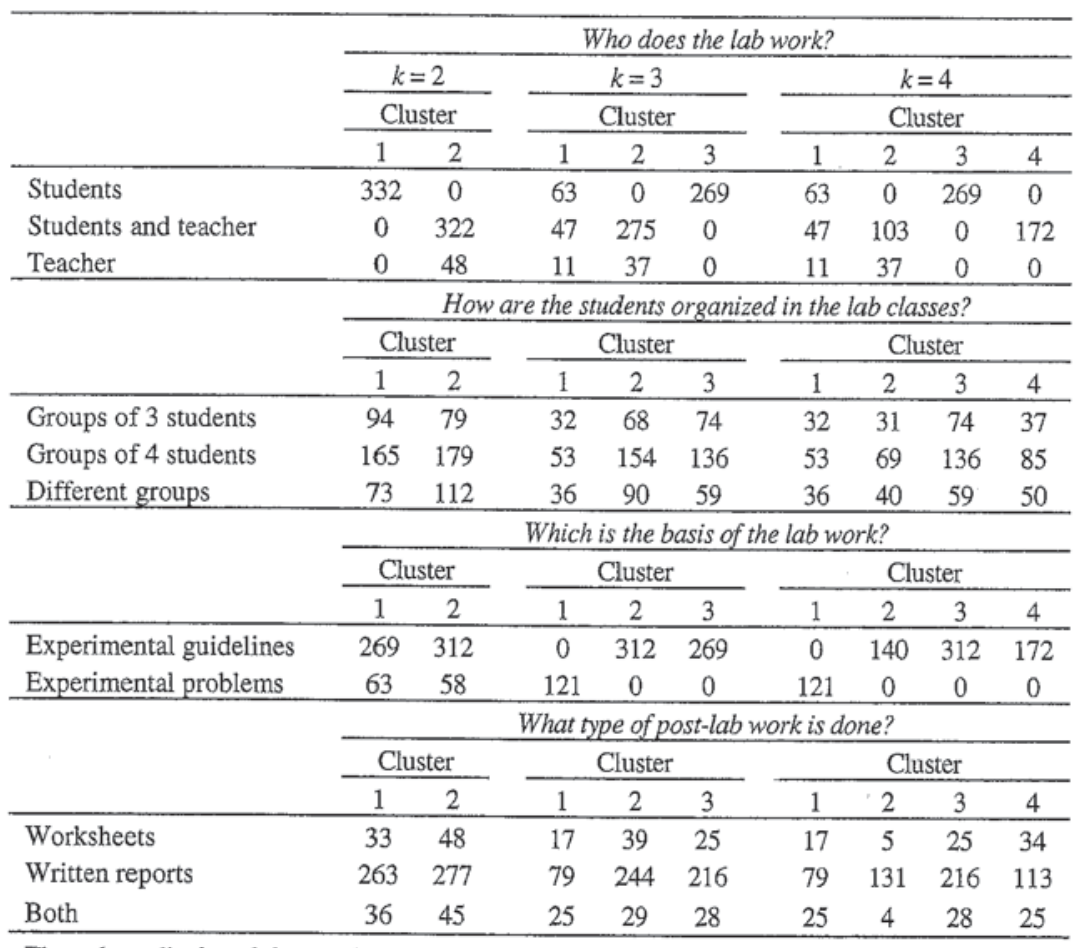

The values displayed denote the average of 20 (twenty) runs 


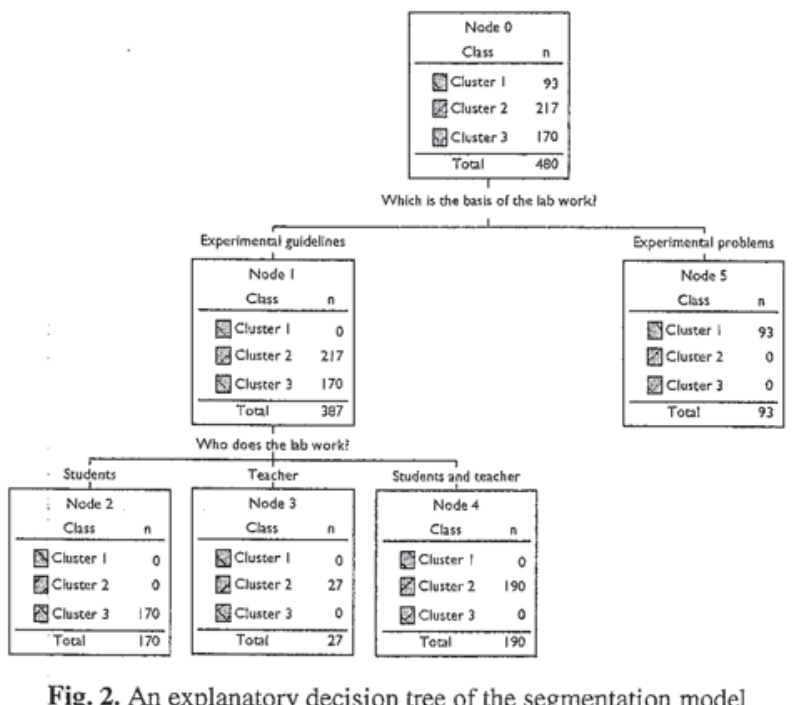

Table 3. The coincidence matrix created by matching the clusters predicted by the model (rows) with the actual clusters (columns)

\begin{tabular}{|c|c|c|c|c|c|c|}
\hline & \multicolumn{3}{|c|}{ Training set } & \multicolumn{3}{|c|}{ Test set } \\
\hline & Cluster 1 & Cluster 2 & Cluster 3 & Cluster 1 & Cluster 2 & Cluster 3 \\
\hline Cluster 1 & 93 & 0 & 0 & 28 & 0 & 0 \\
\hline Cluster 2 & 0 & 217 & 0 & 0 & 95 & 0 \\
\hline Cluster 3 & 0 & 0 & 170 & 0 & 0 & 99 \\
\hline
\end{tabular}

The values displayed denote the average of 20 (twenty) runs

\section{Discussion}

In order to evaluate the influence of the teaching methodology followed in the lab classes on the weight attributed by the answerers to experimentation in Chemistry, the graph presented in Fig. 3 was conceived. The strength of the relationships between clusters and answers is visible on the type of connections. It shows that regardless of the cluster to which the answerers are assigned, the majority of applicants consider that the significance of experimentation in Chemistry learning is Very high or High. Other possible answers like Moderate, Low or Very low are negligible, once to them are assigned less than $2 \%$ of answers. However, a further analysis shows that the highest percentage of answerers, assuming that the significance of experimentation in Chemistry learning is Very high, belongs to cluster 1 (one), i.e., the answerers that state that lab classes are based on tentative situations form this cluster. Another interesting point is related to the fact that no answerers allocated in cluster 1 (one) have endorsed the responses Very low or Low. Only a small percentage $(\leq 0.5 \%)$ answered Moderate. Concerning cluster 2 (two), based on the answerers whose lab classes are built on experimental guidelines and the lab work is not done exclusively by the students, the percentage of most positive responses (Very high and High) are quite similar, and the percentage of less positive and negative responses (Moderate, Low and Very low) is higher than in the other clusters. Regarding cluster 3 (three), that includes the answerers whose lab classes are based on experimental guidelines and the lab work is done exclusively by the students, the results are similar to that obtained for cluster 1 (one) in terms of the positive responses, although exhibiting lesser percentages.

This result may be related with the development of higher level skills associated with the inquiry and the planning of the lab work, which are not present in the lab classes based on experimental guidelines. According Hofstein [26], the appropriate laboratory activities can be effective in promoting cognitive skills, metacognitive skills, practical skills, and attitude and interest towards chemistry, learning chemistry, and practical work in the framework of chemistry learning.

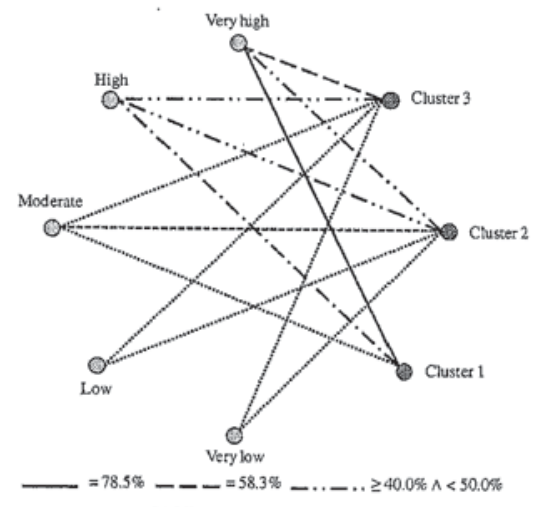

$-16.5050$

Fig. 3. Relationships between clusters and the percentages of answers to questions like Which is the importance of experimentation in the learning of Chemistry?

\section{Conclusions}

In section 3.1 some preliminary results, based on the analysis of the frequencies of the answers given by the applicants in this study, were built. This type of analysis showed that the overwhelming majority of the answerers claim that experimentation is important for Chemistry learning. However, this analysis was unable to discriminate the opinions expressed by the answerers according to the type of the teaching methodology used in the lab classes. Therefore, it was not possible to conclude about the methodology that, in the opinion of the answerers, promotes Chemistry learning and contributes to increase the student's motivation to study this subject. 
The data mining approach using $\mathrm{k}$-means clustering models presented in this study, allowed a deeper analysis of the results (sections 4.1 and 4.2). Indeed, this method of analysis enabled one to identify the methodology to teach Chemistry that leads to a more effective learning.

The encouraging results obtained in this work show that Artificial Intelligence based tools can be very useful to improve the teaching methodologies in order to optimize the teaching and the learning of Chemistry or other subjects.

Acknowledgements. This work is funded by National Funds through the FCT - Fundação para a Ciência e a Tecnologia (Portuguese Foundation for Science and Technology) within projects PEst-OE/EEI/UT0752/2014 and PEst-OE/QUT/UI0619/ 2012.

\section{References}

1. Breuer, S.: Does chemistry have a future? University Chemistry Education 6, 13-16 (2002)

2. Johnstone, A.H.: The development of chemistry teaching: A changing response to changing demand. Journal of Chemical Education 70, 701-705 (1993)

3. Gee, B., Clackson, S.G.: The origin of practical work in the english school science curriculum. School Science Review 73, 79-83 (1992)

4. Hofstein, A., Lunetta, V.N.: The laboratory in science education: foundations for the twenty-first century. Science Education 88, 28-54 (2004)

5. Hofstein, A., Mamlok-Naaman, R.: The laboratory in science education: The state of the art. Chemistry Education Research and Practice 8, 105-107 (2007)

6. Josephsen, J.: Experimental training for chemistry students: does experimental experience from the general sciences contribute? Chemistry Education Research and Practice 4, 205218 (2003)

7. Lock, R.: A history of practical work in school science and its assessment, 1860-1986. School Science Review 70, 115-119 (1988)

8. Miguéns, M., Garrett, R.M.: Prácticas en la enseñanza de las ciencias. Problemas y Posibilidades 9, 229-236 (1991)

9. Millar, R.: The role of practical work in the teaching and learning of science. In: High School Science Laboratories: Role and Vision, p. 24. National Academy of Sciences, Washington DC (2004)

10. Woolnough, B.: Setting the Scene. In: Woolnough, B. (ed.) Pratical Science: The Role and Reality of Practical Work in School Science, pp. 3-9. Open University Press, Milton Keynes (1991)

11. Hodson, D.: A critical look at practical work in school science. School Science Review 70 $33-40$ (1990)

12. Hodson, D.: Re-thinking old ways: Towards a more critical approach to practical work in school science. Studies in Science Education 22, 85-142 (1993)

13. Johnstone, A.H., Al-Shuaili, A.: Learning in the laboratory: Some thoughts from the literature. University Chemistry Education 5, 42-51 (2001)

14. Logar, A., Savec, V.F.: Students' hands-on experimental work vs lecture demonstration in teaching elementary school chemistry. Acta Chimica Slovenica 58, 866-875 (2011)
15. Killerman, W.: Biology education in Germany: research into the effectiveness of different teaching methods. International Journal of Science Education 18, 333-346 (1996)

16. Bennett, J., Hogarth, S., Lubben, F., Campbell, B., Robinson, A.: Talking science: The research evidence on the use of small group discussions in science teaching. International Journal of Science Education 32, 69-95 (2010)

17. Cheung, D.: Facilitating chemistry teachers to implement inquiry-based laboratory work. International Journal of Science and Mathematics Education 6, 107-130 (2008)

18. Witten, I.H., Frank, E.: Data Mining - Practical Machine Learning Tools and Techniques. Elsevier, San Francisco (2005)

19. Han, J., Kamber, M.: Data Mining: Concepts and Techniques. Morgan Kauffmann Publishers, San Francisco (2006)

20. Cohen, L., Manion, L., Morrison, K.: Research Methods in Education. Routledge, New York (2011)

21. DeKetele, J., Roegiers, X.: Méthodologie du Recueil d'Informations: Fondements des Méthodes d'Observation, de Questionnaire, d'Interview et d'Études de documents. DeBoeck Universite, Paris (2009)

22. McMillan, J., Schumacher, S.: Research in Education: Evidence-Based Inquiry. Prentice Hall, New York (2009)

23. Bell, J.: Doing your research project: A guide for first-time researchers in education, health and social science. Open University Press, Maidenhead (2010)

24. Bradley, P.S., Fayyad, U.M.: Refining Initial Points for K-Means Clustering. In: Shavlik, J. (ed.) 15th International Conference on Machine Learning (ICML 1998), pp. 91-99. Morgan Kaufmann, San Francisco (1998)

25. Hall, M., Frank, E., Holmes, G., Pfahringer, B, Reutemann, P., Witten, LH. The WEKA Data Mining Software: An Update. SIGKDD Exploration 11, 10-18 (2009)

26. Hofstein, A.: The laboratory in chemistry education: Thirty years of experience with developments, implementation and evaluation. Chemistry Education Research and Practice 5, 247-264 (2004) 